\title{
Stabilization of Broad Area Semiconductor Laser Sources
}

\author{
J. Medina ${ }^{1}$, W. W. Ahmed ${ }^{12^{*}}$, S. Kumar ${ }^{1}$, M. Botey ${ }^{1}$, R. Herrero ${ }^{1}$, and K. Staliunas ${ }^{1,3}$ \\ ${ }^{I}$ Departament de Física, Universitat Politècnica de Catalunya (UPC), \\ Colom 11, E-08222 Terrassa, Barcelona, Spain \\ ${ }^{2}$ European Laboratory for Non-linear Spectroscopy (LENS), Sesto Fiorentino 50019, Florence, Italy \\ ${ }^{3}$ Institució Catalana de Recerca i Estudis Avançats (ICREA), \\ Passeig Lluís Companys 23, E-08010, Barcelona, Spain \\ e-mail: judithmp93@gmail.com
}

\begin{abstract}
We numerically proof the stabilization of broad area semiconductor laser sources by introducing simultaneous in-phase two-dimensional modulations on the refractive index and on the pump (gain). We also examine the interplay between the index and gain modulations and the effect of the slow relaxation of carriers on the stability performance.
\end{abstract}

Keywords: broad area semiconductor, modulation instability, gain-loss, nanophotonics, stabilization.

\section{INTRODUCTION}

Broad Area Semiconductor (BAS) amplifiers and lasers are promising and reliable light sources, used in many applications due to their compactness and high conversion efficiency. However, a major drawback of such devices is the relatively low spatial and temporal quality of the emitted beam [1,2]. Modulation instability (MI) $[3,4]$ is the fundamental phenomenon that induces transverse mode instabilities, breaking up the mode profile into multiple filaments. Filamentation relies on the dependence of the refraction index on the population inversion [5]. Moreover, strong nonlinear interactions of the optical field with the active media lead to complex spatiotemporal dynamics [6]. Subsequently, the operation of high-power lasers sources is sharply degraded in terms of functionality and fiber coupling. Accordingly, an efficient and compact technique to reduce and control the instability is highly desired.

Recently, an interesting solution was proposed, [7] based on introducing a periodic spatiotemporal modulation of the potential to manipulate and control instabilities in spatially extended nonlinear dynamical systems [8]. The method has shown an improvement in the emission of BAS amplifiers and flat mirror VECSELs $[9,10]$. Although, the role of carrier properties and temporal instabilities was not discussed for BAS amplifiers stabilization.

In this short letter, we consider a model for the BAS laser source that includes the dynamics of carrier density and carrier diffusion, based on [12-13]. We study the system dynamics and its stabilization by introducing simultaneous and in-phase two-dimensional (2D) pump and index modulations. We numerically show the proposed stabilizing mechanism for BAS laser sources, including the effect of slow relaxation and diffusion of the carrier density on the proposed mechanism

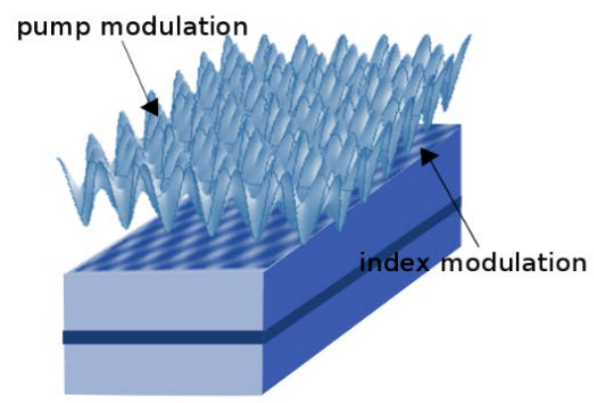

Figure 1. Schematic illustration of a modulated BAS source. The pump (gain/loss) modulation can be achieved by structuring the electrodes and the index modulation by structuring the medium.

The paraxial model describing the dynamics of electric field amplitude $(A)$ and carrier density $(N)$ for the proposed doubly modulated BAS laser source may be expressed as:

$$
\begin{aligned}
& \frac{\partial \mathbf{A}}{\partial z}=\frac{\mathrm{i}}{2 \mathrm{k}_{0} \mathrm{n}} \frac{\partial^{2} \mathbf{A}}{\partial x^{2}}+s[(1-\mathrm{i} h) \mathbf{N}-(1+\alpha)] \mathbf{A}+4 \mathrm{im}_{2}(x, z) \mathrm{k}_{0} \\
& \frac{\partial \mathbf{N}}{\partial t}=\gamma\left(\mathbf{N}-(\mathbf{N}-1)|\mathbf{A}|^{2}+\mathrm{p}+4 \mathbf{m}_{\mathbf{1}}(x, z)+\mathrm{D} \nabla^{2} \mathbf{N}\right)
\end{aligned}
$$

where $n$ is the effective refractive index of the semiconductor, $D$ is the carrier diffusion, $\gamma$ is the carriers relaxation rate, $\alpha$ corresponds to losses and $h$ is the Henry factor or linewidth enhancement factor of the 
semiconductor. The s parameter is inversely proportional to the light matter interaction length and $p$ is the pump parameter for Broad Area Semiconductor (BAS) light sources [11]. The in-phase spatial modulations, $m_{1,2}(x, z)=m_{1,2} \cos \left(q_{x} x\right) \cos \left(q_{z} z\right)$, have the same spatial profile with $q_{x}$ transverse and $q_{z}$ longitudinal wavenumbers and correspond to pump and refractive index modulation with amplitudes $m_{1}$ and $m_{2}$, respectively. The spatial modulations are assumed in the transverse and longitudinal directions on small spatial scales, i.e. $\left|q_{x}\right|>>\left|k_{x}\right|$ and $\left|q_{z}\right|>>|\lambda|$, where $k_{x}$ and $\lambda$ are the typical transverse wavevector and exponential instability growth parameter. The spatial modulation is characterized by the geometrical parameter: $Q=2 n k_{0} q_{z} / q_{x}^{2}$ relating the wavenumbers $q_{x}=2 \pi / d_{\perp}$ and $q_{z}=2 \pi / d_{\|}$being $d_{\perp}$ and $d_{\|}$the transverse and longitudinal period of the modulation, respectively.

\section{RESULTS}

We perform the study of the semiconductor laser stabilization in two complementary ways. From one side we use a direct numerical integration of the unmodulated BAS laser model (Eq. 1) to localize the different dynamical behaviours in the parameter space. The system shows stable homogeneous solutions and chaotic or turbulent behaviours born from the Modulation Instability (Figs. 2a, 2b). The width of the field spectrum indicates the unstable wavenumber range (Fig. 2b).

(a)

(c)
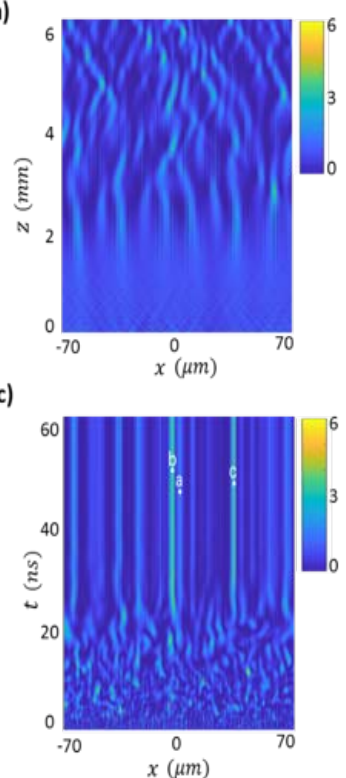

(e)

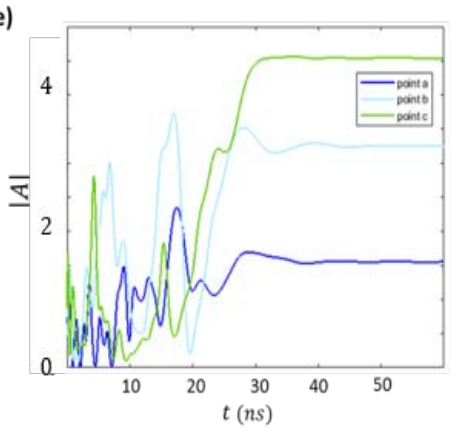

(b)

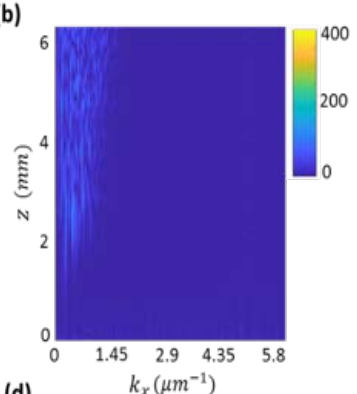

(d)

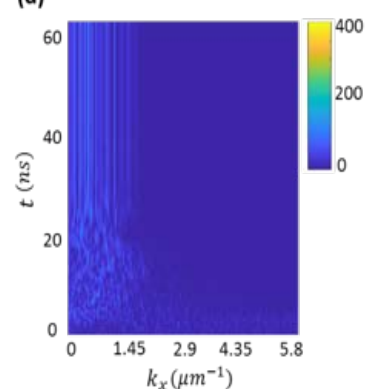

(f)

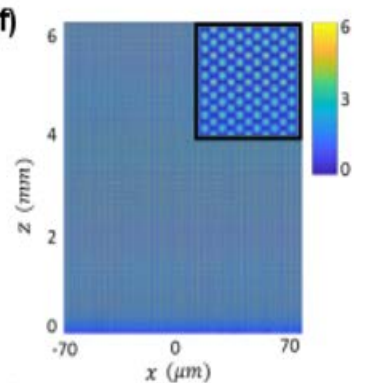

(h)
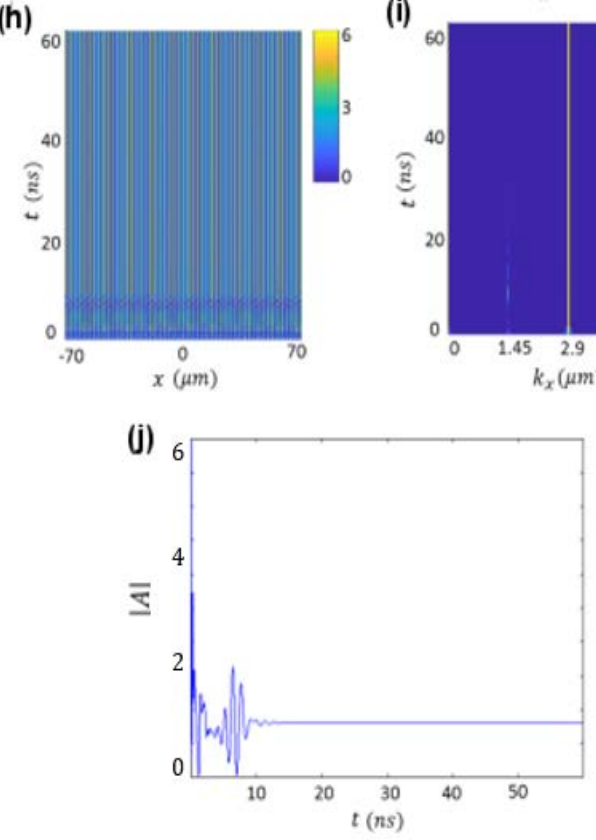

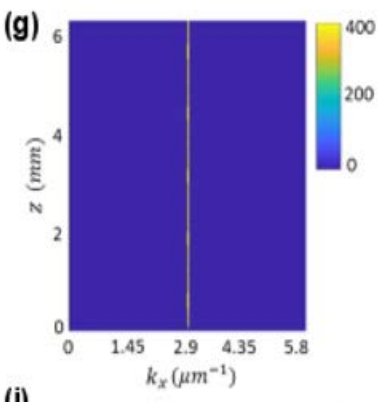

(i)

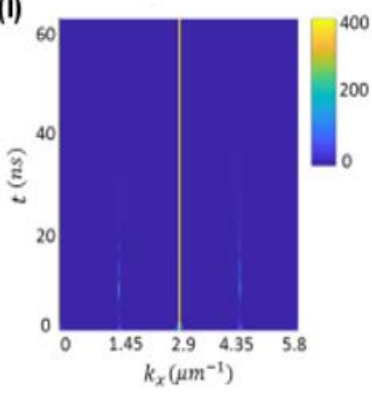

Figure 2: (a-e) Numerical integration for the no modulated case $m_{1}=m_{2}=0$; (a) Field transverse distribution and (b) Spatial spectrum inside the semiconductor after a long integration time. Temporal evolution of (c) field and (d) spectrum at the end of the semiconductor $(z=6 \mathrm{~mm})$. (e) Field temporal evolution at points a,b,c indicated in (c). (f-j) Integration of the pump and index modulated case $m_{1}=0.35 ; m_{2}=0.001$; (f) Field distribution and (g) Spatial spectrum inside the semiconductor after a long integration time. Temporal evolution of $(h)$ field and (i) spectrum at the end of the semiconductor. (j) Time evolution of the field at coordinates $(x=0 \mathrm{~mm}, z=6 \mathrm{~mm})$. The simulation parameters are width $140 \mu \mathrm{m}$, length $6.4 \mathrm{~nm}$, refractive index 3.3 , carrier diffusion $0.15 \mathrm{~cm}^{2} / \mathrm{s}$, linewidth enhancement factor $h=2.0$, normalized losses $\alpha=0.1 \mu^{-1}$, inverse of light matter interaction length $s=$ $0.03 \mathrm{\mu m}^{-1}$, normalized carrier relaxation rate $\gamma=0.01$, pumping intensity $p=1.21 \mathrm{~A}$, transverse modulation wavenumber $q_{x}=2.852 \mu \mathrm{m}^{-1}$ and longitudinal modulation wavenumber $q_{z}=0.1965 \mu \mathrm{m}^{-1}$. 
However, the considered model, allows the analysis of temporal features and for some parameter sets, the turbulent system appears stable in time. The field shows a highly inhomogeneous distribution in transverse space and in temporal propagation (Figs. $2 \mathrm{c}-2 \mathrm{~d}$ ). The field stabilization after a long transient time of $\approx 40 \mathrm{~ns}$ is shown by the temporal evolution of the three points marked as (a, b, c) in (Fig. 2c) and plotted in (Fig. 2e).

We study the laser source stabilization by introducing spatial modulations of pump and refractive index). A partial stabilization is obtained by the introduction of only a pump or a refractive index modulation while the complete stabilization is achieved by the simultaneous modulation of pump and refractive index, see the numerical results presented in (Figs. $2 \mathrm{f}-2 \mathrm{j}$ ). The result shows the full stabilization in the field propagation and also in temporal evolution. For this case, the spatial spectrum $A\left(k_{x}\right)$ is only composed by transverse modulation wavenumber, $q_{x}$, proving the total stabilization. This could be also seen in the inset in (Fig. 2f), where the modulation appears to be perfectly homogeneous.

The temporal evolution of the transvers spatial distribution becomes totally stabilized under both modulations (Figs. 2h-2i). The temporal transient becomes shorter as compared to the previous case, with a duration on the order of 15 ns up to obtain the desired temporally stable homogeneous solution.

We characterize the doubly-modulated laser source in the parameter space and localize both associated bifurcations, the Modulation Instability related with the arousal of spatial instabilities, spatially chaotic field distributions and filamentation, and the Hopf bifurcation associated to the temporal instability of the homogeneous solution.

\section{CONCLUSIONS}

We numerically prove that simultaneous 2D modulations of pump and refractive index provide an efficient scheme to stabilize BAS laser sources, both in space and time. We perform numerical simulations on the full paraxial model considering field and carriers with realistic parameters and the results confirm that the doublymodulated configuration can completely suppress Modulation Instabilities and Hopf instabilities in the homogeneous solution and offers a flexible control on spatiotemporal dynamics of BAS devices.

\section{REFERENCES}

[1] O. Hess, S. W. Koch, and J. V. Moloney: Filamentation and beam propagation in broad area semiconductor lasers, IEEE J. Quantum Electron., 31, 35, 1995.

[2] J. Marciante and G. Agrawal: Nonlinear mechanisms of filamentation in broad-area semiconductor lasers, IEEE J. Quantum Electron., 32, 590, 1996.

[3] F. Prati and L. Columbo: Long-wavelength instability in broad-area semiconductor lasers, Rev. A., 75, $053811,2007$.

[4] V. I. Bespalov and V. I. Talanov: Self-focusing of inhomogeneous laser beams and its effect on stimulated scattering, JETP Lett., 3, 307, 1966.

[5] S. Wolff and H. Fouckhardt: Intracavity stabilization of broad area lasers by structured delayed optical feedback, Opt. Express 7, 222-227, 2000.

[6] H. Adachihara, O. Hess, E. Abraham, and J. V. Moloney: Semiconductor laser array dynamics: numerical simulations on multistripe index-guided lasers, J. Opt. Soc. Am. B., 10, 496, 1993.

[7] W. Ahmed, J. Medina, R. Herrero, M. Botey, and K. Staliunas: Stabilization of broad area semiconductor laser sources by simultaneous index and pump modulations, submitted to Opt. Lett.

[8] S. Kumar, R. Herrero, M. Botey, and K. Staliunas: Suppression of modulation instability in broad area semiconductor amplifiers, Opt. Lett. 39, 5598-5601, 2014.

[9] W. Ahmed, S. Kumar, R. Herrero, M. Botey, M. Radziunas and K. Staliunas: Stabilization of flat-mirror vrtical-external-cavity surface-emitting lasers by spatiotemporal modulation of the pump profile, Phys. Rev. A 92, 04382, 2015.

[10] W. Ahmed, R. Herrero, M. Botey, and K. Staliunas: Locally parity-time-symmetric and globally paritysymmetric systems, Phys. Rev. A., 94, 053819, 2016.

[11] M. Osinski and J. Buus: Linewidth broadening factor in semiconductor lasers, IEEE J. Quantum Electron., 23, 9, 1987.

[12] G. P. Agrawal: Fast-Fourier-transform based beam-propagation model for stripe-geometry semiconductors lasers: Inclusion of axial effects, J. Appl. Phys., 56, 3100, 1984.

[13] E. A. Ultanir, D. Michaelis, F. Lederer, and G. I. Stegeman: Stable spatial solitons in semiconductor optical amplifiers, Opt. Lett., 28, 251, 2003. 